\title{
Factores de riesgo en trastornos de la conducta alimentaria en deportistas de alto rendimiento. Revisión sistemática
}

\section{Risk factors for eating disorders in high performance athletes. Systematic review}

\author{
Alba Villamediana Sáez y José Ignacio Baile Ayensa \\ Universidad a Distancia de Madrid, España
}

\begin{abstract}
Resumen: Introducción. Diferentes estudios destacan una importante prevalencia de los Trastornos de la Conducta Alimentaria en el contexto del Alto Rendimiento Deportivo, sin embargo, no se han estudiado con detalle las causas de ello. Objetivo. Estudiar los diferentes factores que parecen contribuir al desarrollo de TCA, o proteger, en este grupo de deportistas, teniendo en cuenta las características del alto rendimiento y los diferentes tipos de deporte. Metodología. Se ha realizado una revisión sistemática de artículos publicados en PsycINFO, Dialnet, SciELO, PubMed y Google Scholar, que analizaran posibles factores de riesgo o protectores de TCA. Resultados. Tras descartar los artículos no elegibles, se seleccionaron de 23 artículos que cumplían los requisitos para la revisión. Conclusiones. La influencia de las presiones por el resultado deportivo, la relación de éste con el peso corporal o la cultura deportiva aparecen como factores de riesgo, así como la importancia de la información nutricional y la psicoeducación en deportistas, entrenadores y personas de influencia como factores protectores. Limitaciones: Estos resultados no ofrecen una relación causal, por lo que se requiere mayor investigación de las relaciones entre variables.

Palabras clave: trastorno conducta alimentaria, deportista alto rendimiento, factores de riesgo, factores de protección, revisión.
\end{abstract}

Abstract: Different studies report a significant prevalence of eating disorders in high sports performance, but the reasons for such prevalence have not been studied in detail. Objective. To study the different factors that appear to contribute to or to protect from

Alba Villamediana Sáez es Psicóloga General Sanitaria y del Deporte.

José Ignacio Baile Ayensa es Doctor en Psicología; Doctor en Derecho y Sociedad, Profesor Titular del Grado de Psicología en la Universidad a Distancia de Madrid UDIMA.

Para citar este artículo: Villamediana, A. y Baile, J. I. (2021). Factores de riesgo en trastornos de la conducta alimentaria en deportistas de alto rendimiento. Revisión sistemática. Clínica Contemporánea, 12(2), Artículo e13. https://doi.org/10.5093/cc2021a9

La correspondencia sobre este artículo deberá ser enviada a José Ignacio Baile. E-mail: ignaciobaile@ hotmail.com

(cc) BY-NC-ND Este es un artículo Open Access bajo la licencia CC BY-NC-ND. 
the development of eating disorders, in this group of athletes, taking into account the characteristics of high performance and the different types of sport. Method. A systematic review was conducted of articles published in PsycINFO, Dialnet, SciELO, PubMed and Google Scholar analyzing possible risk or protective factors as regards eating disorders. Results. After ruling out ineligible articles, 23 articles that met the requirements for review were selected. Conclusions. The influence of the pressures for the sporting result, the relationship of the result with body weight or the sports culture appear as risk factors, while the importance of nutritional information and psychoeducation in athletes, coaches, and influential people appeared as protective factors. Limitations. These results do not provide a causal relationship, and further research on the relations between variables is therefore required.

Keywords: eating disorder, high performance athletes, risk factors, protective factors, review

Numerosos estudios han relacionado la práctica de actividad física con una mayor satisfacción con la imagen corporal, el establecimiento de hábitos saludables, y la disminución de factores de riesgo para la salud relacionados con el sedentarismo como son la obesidad, el colesterol o estados anímicos depresivos (De Sá et al., 2017).

Sin embargo, esta relación positiva no se produce en todos los ámbitos, ya que la práctica deportiva excesiva puede conllevar algunos riesgos físicos, como el excesivo control del peso, sobreentrenamiento o la aparición de lesiones, y mentales como los problemas de autoimagen o los Trastornos de la Conducta Alimentaria (TCA) (Cano y Fernández, 2017; Díaz y Dosil, 2012; Fabra y Casadó, 2014; Ghoch et al., 2013; Karrer et al., 2020).

Tal y como afirmaban Cano y Fernández (2017), la realización excesiva de actividad física es un síntoma común que afecta hasta un $80 \%$ de los pacientes con TCA, sin embargo, esta característica no puede ser tenida en cuenta en deportistas de alto rendimiento (DAR en adelante), puesto que la gran cantidad de horas de entrenamiento es inherente a la alta competición. De hecho, muchos atletas adoptan un comportamiento alimentario típico de los pacientes diagnosticados con TCA, como restringir la ingesta de alimentos durante largos períodos o el control diario de peso, que en pacientes no deportistas serían señales de alarma de TCA (De Sousa et al., 2016).

\section{Prevalencia}

Según un estudio de Dosil (2003), los casos de TCA han aumentado significativamente en los últimos años en el ámbito del alto rendimiento, estimando la incidencia de TCA entre un 4.2 y el 39.2 \%. En comparación con personas no deportistas, encontramos un estudio realizado por Sundgot-Borgen y Torstveit (2004), el cual demostró que la prevalencia de TCA en esta población era de $13.5 \%$, frente al $3.4 \%$ de los no deportistas, datos que corroboró un estudio por rangos de edad de Pérez et al. (1992) el cual indicó que los atletas de alto rendimiento de entre 14 y 25 años de los equipos nacionales, el 13\% podría tener TCA, mientras que la tasa entre las personas sedentarias fue del 5.5\%. También hay que mencionar la diferencia de prevalencia entre las mujeres deportistas y los hombres, siendo ésta de un $34.5 \%$ frente a un $16.7 \%$ en los hombres. Hay que tener en cuenta que estos porcentajes varían según el tipo de deporte; los deportes denominados "de riesgo" la prevalencia de un TCA fue tres veces mayor (36\%) que en otras modalidades deportivas (13\%) (Martínez, et al., 2012). En este tipo de deportes con mayor riesgo, según mencionaba Franchini et al. (2012), el uso de estrategias rápidas de pérdida de peso (como uso de laxantes, restricción calórica severa, deshidratación, etc.) se practica entre el 75 y el $90 \%$ de los atletas de nivel internacional y nacional. 


\section{TCA en Deportistas de Alto Rendimiento, factores asociados}

En sintonía con el último dato expuesto sobre la prevalencia, Perez et al. (1992) expuso en su investigación que los atletas de estos deportes denominados "de riesgo" tienen una mayor probabilidad de relacionarse con los TCA que otros deportes. Este grupo denominado "de riesgo" se encontraban aquellos que, por normativa, hay una separación de los deportistas por categorías de peso corporal, o bien aquellos en los que es preferible un bajo peso o una apariencia corporal delgada por razones estéticas. También entran en dicho grupo los deportes en donde el rendimiento del atleta se evalúa subjetivamente (con jueces o árbitros), o los denominados de resistencia aeróbica (Goltz, et al., 2013; Martínez et al., 2012).

El estudio llevado a cabo por Díaz et al. (2018), tuvo como objetivo era determinar, a través del cuestionario de hábitos alimentarios del atleta (CHAD), si existen realmente factores de riesgo psicosocial para los TCA entre atletas de diferentes deportes $(\mathrm{N}=357)$. Dichos autores encontraron que en deportes en los que la forma o el peso corporal se consideran menos importantes, como por ejemplo el fútbol, las atletas femeninas aún son más propensas a desarrollar TCA que las que no son atletas.

Además de las características inherentes del propio deporte, las características de personalidad influyen en el desarrollo del TCA, observando a menudo un perfeccionismo adaptativo, el cual se asocia con un mayor rendimiento en entrenamientos, mientras que se ignora los peligros de un perfeccionismo desadaptativo (Prnjak et al., 2019).

Otro factor relevante en relación a los TCA en deportistas de alto rendimiento se asocia con la identidad deportiva. Según decía Packard (2010), la participación deportiva exige elevados niveles de compromiso, y para los DAR termina siendo la actividad central de su vida diaria, pudiendo llegar a fundamentar su propia identidad personal en su identidad deportiva (ID). Tras un estudio realizado por Hernández-Mulero y Berengüí, (2016) en el que se administra a 109 deportistas federados la versión traducida del AIMS (Athletic Identity Measurement Scale) de Mateos et al. (2010), se vio que una elevada ID puede conllevar efectos nocivos, como alta dependencia de la autoestima al resultado, formación de expectativas irreales, presión por alcanzar el éxito, etc.

\section{Objetivo e hipótesis}

Debido a todos los datos comentados, en el presente trabajo tiene como objetivo estudiar los factores de riesgo y de protección que relacionan los TCA con los deportistas de alto rendimiento según las evidencias existentes en la literatura, con el fin de poder elaborar líneas de prevención que disminuya la incidencia de este trastorno en el deporte de élite.

Para ello partimos con unas hipótesis extraídas tras la revisión inicial de la literatura, que nos ayudarán y nos guiarán en la búsqueda sistemática:

- Hipótesis 1: El deporte de alto rendimiento cuenta con factores de riesgo intrínsecos a la propia cultura del deporte que hace que aumente el riesgo a padecer un TCA.

- Hipótesis 2: El alto rendimiento deportivo tiene consecuencias negativas en la percepción de la imagen corporal.

- Hipótesis 3: Existen factores de protección que se deben tener en cuenta en el alto rendimiento que favorecen la prevención del TCA.

- Hipótesis 4: Una buena relación atleta-entrenador, compañeros y familiares actúa como factor de protección frente a la posibilidad de padecer un trastorno de alimentación en alto rendimiento.

\section{Método}

La revisión sistemática del presente trabajo, siguiendo las líneas principales de la guía PRISMA, se realizó en noviembre de 2019 empleando bases de datos de referencia en la psicología (PsycINFO, Dialnet, SciE- 
LO, PubMed y Google Scholar). Para ello se utilizó una metodología cualitativa de selección y de búsqueda de artículos por palabras claves (tabla 1), y ajustando los criterios de inclusión y exclusión detallados en la tabla 2 .

Tabla 1. Artículos incluidos y excluidos tras la búsqueda sistemática

\begin{tabular}{ll}
\hline Base de datos & Palabras clave utilizadas \\
\hline Dialnet & $\begin{array}{l}\text { Trastorno alimentación AND deporte alto rendimiento } \\
\text { Trastorno conducta alimentaria AND deporte }\end{array}$ \\
\hline Google académico & "TCA" AND "deporte alto rendimiento" \\
\hline PubMed & Eating disorders high-performance sports \\
\hline PsychINFO & Eating disorders high-performance sports \\
\hline Scielo & Eating disorders high-performance sports \\
& Eating disorders sports \\
\hline
\end{tabular}

Tabla 2. Criterios de inclusión y exclusión para el presente trabajo de revisión sistemática

\begin{tabular}{|c|c|}
\hline Criterios de inclusión & Criterios de exclusión \\
\hline Antigüedad máxima 10 años & Antigüedad mayor de 10 años \\
\hline 2009- 2019 & $<2009$ \\
\hline Cualquier deporte de alto rendimiento & Nivel de práctica deportiva por debajo al alto rendimiento \\
\hline Individual & Deporte base \\
\hline \multirow[t]{3}{*}{ Equipo } & Tecnificación \\
\hline & Deporte Amateur \\
\hline & Ocio-deporte \\
\hline Trastornos de Conducta Alimentaria & Trastornos de Conducta Alimentaria \\
\hline Anorexia Nerviosa & Trastorno Dismórfico Corporal \\
\hline Bulimia Nerviosa & Trastorno por atracón \\
\hline \multirow{5}{*}{$\begin{array}{l}\text { Español } \\
\text { Inglés } \\
\text { Portugués } \\
\text { Francés }\end{array}$} & Idioma \\
\hline & Resto de idiomas no citados \\
\hline & \\
\hline & \\
\hline & \\
\hline
\end{tabular}

De la búsqueda con los criterios específicos se partió con un total de 96 artículos, de los cuales se realizó un primer cribado de los artículos duplicado (eliminando 2). Posteriormente se eliminaron otros 25 artículos por criterios de idioma y año de publicación. Tras la lectura de los abstract se seleccionaron los 23 artículos finales de los cuales se extraerían los resultados que se presentan en el presente documento (figura 1). 
Figura 1. Algoritmo de búsqueda sistemática

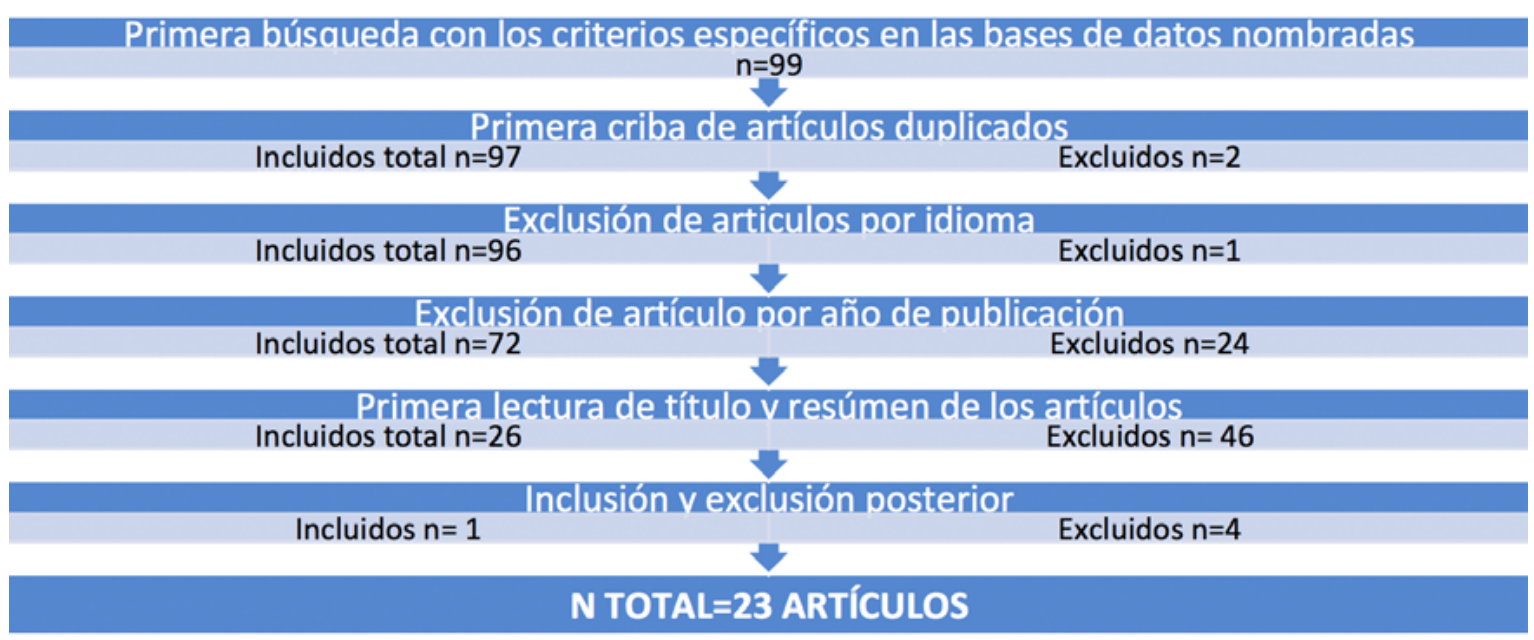

Análisis de datos

Tras la lectura detallada de los artículos incluidos en la búsqueda sistemática se realizó un cuadro resumen de los resultados obtenidos por los autores, clasificándolo bajo la etiqueta de "factor de riesgo" o "factor de protección".

Como factores de riesgo se siguió la clasificación descrita por la Organización Mundial de la Salud (OMS) en el que lo define como cualquier rasgo, característica o exposición de un individuo que aumente su probabilidad de sufrir una enfermedad o trastorno. Por otro lado, dentro del campo de los factores de protección se abarca todo aquello que puede disminuir la probabilidad de contraer cierta enfermedad o trastorno.

\section{Resultados}

El número total de artículos analizados fueron de 23 de los cuales 6 de ellos eran revisiones bibliográficas y los restantes estudios experimentales, en la tabla 3 se recogen dichos artículos y sus datos principales.

Tabla 3. Resumen de los resultados obtenidos.

\begin{tabular}{|c|c|c|c|c|c|}
\hline $\begin{array}{l}\text { AUTORES DEL } \\
\text { ESTUDIO }\end{array}$ & AÑO & OBJETIVO & MUESTRA & FACTORES DE RIESGO & FACTORES DE PROTECCIÓN \\
\hline Cano y Fernández & 2017 & $\begin{array}{l}\text { Revisión sistemática de } \\
\text { casos de anorexia en } \\
\text { deporte }\end{array}$ & 20 artículos & $\begin{array}{l}\text { La búsqueda de peso para mejo- } \\
\text { rar el rendimiento }\end{array}$ & \\
\hline \multirow{6}{*}{$\begin{array}{l}\text { Martínez, Urdam- } \\
\text { pilleta, Micó y So- } \\
\text { riano }\end{array}$} & \multirow[t]{6}{*}{2012} & \multirow{6}{*}{$\begin{array}{l}\text { Conocer las necesida- } \\
\text { des nutricionales de- } \\
\text { pendiendo del deporte }\end{array}$} & \multirow[t]{6}{*}{38 artículos } & $\begin{array}{l}\text { Creencias erróneas sobre la ali- } \\
\text { mentación. }\end{array}$ & $\begin{array}{l}\text { Hábitos alimentarios establecidos du- } \\
\text { rante la infancia. }\end{array}$ \\
\hline & & & & $\begin{array}{l}\text { Perfeccionismo, compulsividad y } \\
\text { altas expectativas sobre sí mismo. }\end{array}$ & \multirow{5}{*}{$\begin{array}{l}\text { Percibir mejora en su rendimiento ante } \\
\text { una alimentación equilibrada. } \\
\text { Información adecuada sobre hábitos } \\
\text { de nutrición. } \\
\text { Asesoramiento profesional de pautas } \\
\text { adecuadas de alimentación }\end{array}$} \\
\hline & & & & $\begin{array}{l}\text { Presión social de los compañeros } \\
\text { de equipo, entrenador, público y la }\end{array}$ & \\
\hline & & & & ejercida por el propio deporte. & \\
\hline & & & & $\begin{array}{l}\text { Necesidad de mantener un peso } \\
\text { para asegurar el rendimiento. }\end{array}$ & \\
\hline & & & & $\begin{array}{l}\text { Conductas incorrectas de los en- } \\
\text { trenadores }\end{array}$ & \\
\hline
\end{tabular}


Tabla 3 (Continuación). Resumen de los resultados obtenidos

\begin{tabular}{|c|c|c|c|c|c|}
\hline $\begin{array}{l}\text { AUTORES DEL } \\
\text { ESTUDIO }\end{array}$ & AÑO & OBJETIVO & MUESTRA & FACTORES DE RIESGO & FACTORES DE PROTECCIÓN \\
\hline $\begin{array}{l}\text { De Bruin, Oudejans, } \\
\text { Bakker y Woertman }\end{array}$ & 2011 & $\begin{array}{l}\text { La estimación del riesgo } \\
\text { a padecer TCA teniendo } \\
\text { en cuenta la sensibili- } \\
\text { dad de la imagen cor- } \\
\text { poral dependiendo del } \\
\text { tipo de deporte }\end{array}$ & 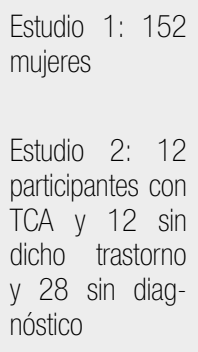 & $\begin{array}{l}\text { Preocupación por la imagen cor- } \\
\text { poral relacionada con el deporte } \\
\text { Comparación social } \\
\text { Disgusto con la propia imagen } \\
\text { corporal presionada por la cultura } \\
\text { deportiva y el rendimiento }\end{array}$ & $\begin{array}{l}\text { Aceptación de las variaciones corpo- } \\
\text { rales } \\
\text { Refuerzo social sobre sus cuerpos }\end{array}$ \\
\hline Díaz y Dosil & 2012 & $\begin{array}{l}\text { Conocer los factores } \\
\text { que influyen en la vul- } \\
\text { nerabilidad y proponer } \\
\text { medidas preventivas } \\
\text { para evitar su aparición }\end{array}$ & 476 sujetos & $\begin{array}{l}\text { Especialización temprana } \\
\text { Doping } \\
\text { Presión social por estar delgado } \\
\text { Presión por conseguir un "cuerpo } \\
\text { ideal" para rendir } \\
\text { Alta tendencia al perfeccionismo } \\
\text { Influencia de los medios de comu- } \\
\text { nicación y periodismo deportivo } \\
\text { Preocupación transmitida por su } \\
\text { entrenador } \\
\text { Comentarios sobre la preocu- } \\
\text { pación por el peso del grupo de } \\
\text { pares } \\
\text { Perfeccionismo, absolutismo, mie- } \\
\text { do al fracaso, rigidez... } \\
\text { Dificultad para gestión emocional } \\
\text { Retirada deportiva } \\
\text { Estar en un centro de alto rendi- } \\
\text { miento deportivo (CARD) } \\
\text { Lesión deportiva }\end{array}$ & $\begin{array}{l}\text { Beneficios a nivel psicosocial e iden- } \\
\text { tidad de grupo relacionados con el } \\
\text { deporte } \\
\text { Sensación subjetiva de "estar en for- } \\
\text { ma" } \\
\text { Educación nutricional de los entrena- } \\
\text { dores }\end{array}$ \\
\hline $\begin{array}{l}\text { Goltz, Stenzel y } \\
\text { Schneider }\end{array}$ & 2013 & $\begin{array}{l}\text { La identificación de TCA } \\
\text { y descontento con la } \\
\text { imagen física en hom- } \\
\text { bres practicantes de } \\
\text { deportes de riesgo para } \\
\text { los TCA }\end{array}$ & $\begin{array}{l}153 \text { hombres } \\
\text { deportistas de } \\
\text { deportes de } \\
\text { combate }\end{array}$ & $\begin{array}{l}\text { Percepción del deporte relaciona- } \\
\text { da con la delgadez } \\
\text { Alta intensidad deportiva con des- } \\
\text { organización en la dieta } \\
\text { Descontento con la propia imagen } \\
\text { corporal }\end{array}$ & $\begin{array}{l}\text { No relación peso y resultado por parte } \\
\text { de los entrenadores }\end{array}$ \\
\hline $\begin{array}{l}\text { De Sá, Aliete dos } \\
\text { Santos y Simões }\end{array}$ & 2017 & $\begin{array}{l}\text { Evaluar el riesgo de de- } \\
\text { sarrollar TCA y los facto- } \\
\text { res que se asocian con } \\
\text { este riesgo entre las mu- } \\
\text { jeres físicamente activas. }\end{array}$ & $\begin{array}{l}356 \text { mujeres de- } \\
\text { portistas }\end{array}$ & $\begin{array}{l}\text { Baja autoestima } \\
\text { Insatisfacción con su cuerpo }\end{array}$ & $\begin{array}{l}\text { Combinación formación académica y } \\
\text { entrenamiento deportivo }\end{array}$ \\
\hline $\begin{array}{l}\text { Lopes, JZotto, Viei- } \\
\text { ra, Fiorese, Cardo- } \\
\text { so y Garcia }\end{array}$ & 2009 & $\begin{array}{l}\text { Ver la relación entre la } \\
\text { distorsión corporal y los } \\
\text { TCA en gimnastas }\end{array}$ & 48 gimnastas & $\begin{array}{l}\text { El peso corporal bajo como requi- } \\
\text { sito para la participación en com- } \\
\text { peticiones deportivas } \\
\text { Peso corporal relacionado con } \\
\text { características relacionadas con } \\
\text { el deporte: flexibilidad, ligereza... }\end{array}$ & $\begin{array}{l}\text { Buenas educación nutricional antes de } \\
\text { la adolescencia }\end{array}$ \\
\hline
\end{tabular}


Tabla 3 (Continuación). Resumen de los resultados obtenidos

\begin{tabular}{|c|c|c|c|c|c|}
\hline $\begin{array}{l}\text { AUTORES DEL } \\
\text { ESTUDIO }\end{array}$ & AÑO & OBJETIVO & MUESTRA & FACTORES DE RIESGO & FACTORES DE PROTECCIÓN \\
\hline $\begin{array}{l}\text { Thiemann, Le- } \\
\text { genbauer, Vocks, } \\
\text { Platen, Auyeung, y } \\
\text { Herpertz }\end{array}$ & 2015 & $\begin{array}{l}\text { La prevalencia de TCA } \\
\text { en jugadoras de depor- } \\
\text { tes de pelota en com- } \\
\text { paración con personas } \\
\text { no deportistas }\end{array}$ & $\begin{array}{l}108 \text { jugadoras } \\
\text { profesionales }\end{array}$ & $\begin{array}{l}\text { La discrepancia entre el cuerpo } \\
\text { adulto y el ideal de competición } \\
\text { La presión por la delgadez en la } \\
\text { cultura deportiva } \\
\text { Especialización temprana }\end{array}$ & $\begin{array}{l}\text { Realizar un deporte de equipo o de } \\
\text { pelota donde el cuerpo adulto y el de- } \\
\text { portivo no discrepan mucho }\end{array}$ \\
\hline $\begin{array}{l}\text { Frideres, Visiedo, y } \\
\text { Palao }\end{array}$ & 2014 & $\begin{array}{l}\text { Evaluar la situación que } \\
\text { se encuentran las juga- } \\
\text { doras profesionales de } \\
\text { voleibol y su percepción } \\
\text { de la situación en rela- } \\
\text { ción al TCA }\end{array}$ & 41 jugadoras & $\begin{array}{l}\text { Medios de comunicación y co- } \\
\text { mentarios sobre imagen física } \\
\text { Estrés competitivo } \\
\text { Falta de información por parte de } \\
\text { los entrenadores y comunicación } \\
\text { con las deportistas }\end{array}$ & $\begin{array}{l}\text { Deportistas formadas en el manejo y } \\
\text { tolerancia al estrés } \\
\text { Fuentes de información adecuadas y } \\
\text { veraces } \\
\text { Abordaje multidisciplinar de la forma- } \\
\text { ción del deportista }\end{array}$ \\
\hline $\begin{array}{l}\text { McArdle, Meade y } \\
\text { Moore }\end{array}$ & 2015 & $\begin{array}{l}\text { Investigar cualitativa- } \\
\text { mente el apoyo pro- } \\
\text { fesional recibido en el } \\
\text { deporte de élite }\end{array}$ & $\begin{array}{l}503 \text { profesiona- } \\
\text { les del ámbito } \\
\text { deportivo }\end{array}$ & $\begin{array}{l}\text { El estigma asociado a los TCA difi- } \\
\text { culta su pronta detección } \\
\text { Sobreprotección y evitación como } \\
\text { mecanismo de afrontamiento } \\
\text { emocional } \\
\text { Nivel de exposición en la vida pú- } \\
\text { blica y redes sociales } \\
\text { Confidencialidad como barrera } \\
\text { para la prevención }\end{array}$ & $\begin{array}{l}\text { La búsqueda de síntomas tempranos } \\
\text { más allá del mero peso corporal } \\
\text { Buena regulación emocional }\end{array}$ \\
\hline De la Fuente & 2018 & & $\begin{array}{l}153 \\
\text { taekwondistas }\end{array}$ & $\begin{array}{l}\text { La necesidad de ajustar un peso } \\
\text { exacto el día de la competición } \\
\text { La sensación de ligereza y dina- } \\
\text { mismo con un peso bajo }\end{array}$ & $\begin{array}{l}\text { La sensación de fortaleza con un peso } \\
\text { más alto }\end{array}$ \\
\hline $\begin{array}{l}\text { Hernández-Mulero } \\
\text { y Berengüí }\end{array}$ & 2016 & $\begin{array}{l}\text { Diferencias en Identidad } \\
\text { Deportiva y TCA entre } \\
\text { hombres y mujeres, y la } \\
\text { posible relación existen- } \\
\text { te entre ambos }\end{array}$ & 109 deportistas & $\begin{array}{l}\text { Medios de comunicación } \\
\text { Moda } \\
\text { Presión del entrenador } \\
\text { Ser mujer } \\
\text { Identidad deportiva alta } \\
\text { Perfeccionismo }\end{array}$ & Apoyo de la familia \\
\hline Loucks & 2014 & $\begin{array}{l}\text { Ver las cuasas de la } \\
\text { baja disponibilidad } \\
\text { energética de los de- } \\
\text { portistas }\end{array}$ & No especificado & $\begin{array}{l}\text { Síntomas de la triada deportiva (la } \\
\text { salud reproductiva y esquelética } \\
\text { se ve afectada por la deficiencia } \\
\text { energética) }\end{array}$ & $\begin{array}{l}\text { Pautas para lograr la energía de forma } \\
\text { correcta }\end{array}$ \\
\hline $\begin{array}{l}\text { Martínez, Pérez, } \\
\text { Santín y Arrue }\end{array}$ & 2018 & $\begin{array}{l}\text { Asemejar los criterios } \\
\text { de DSM-5 y factores } \\
\text { de riesgo ejemplifican- } \\
\text { do con la película Per- } \\
\text { fect Body }\end{array}$ & No especificado & $\begin{array}{l}\text { Ser adolescente } \\
\text { Personalidad perfeccionista } \\
\text { Necesidad de agradar al entorno } \\
\text { Insatisfacción general corporal } \\
\text { Grado de compromiso con su } \\
\text { deporte } \\
\text { Aislamiento del grupo de entrena- } \\
\text { miento }\end{array}$ & Padres concienciados \\
\hline $\begin{array}{l}\text { Prnjak, Jukic y Tu- } \\
\text { fano }\end{array}$ & 2019 & $\begin{array}{l}\text { Evaluar como el per- } \\
\text { feccionismo adaptativo } \\
\text { y no-adaptativo puede } \\
\text { predecir el desarrollo } \\
\text { de TCA }\end{array}$ & $\begin{array}{l}280 \text { deportistas } \\
\text { de deportes in- } \\
\text { dividuales y co- } \\
\text { lectivos }\end{array}$ & $\begin{array}{l}\text { Perfeccionismo } \\
\text { Ser mujer }\end{array}$ & Satisfacción corporal \\
\hline
\end{tabular}


Tabla 3 (Continuación). Resumen de los resultados obtenidos

\begin{tabular}{|c|c|c|c|c|c|}
\hline $\begin{array}{l}\text { AUTORES DEL } \\
\text { ESTUDIO }\end{array}$ & AÑO & OBJETIVO & MUESTRA & FACTORES DE RIESGO & FACTORES DE PROTECCIÓN \\
\hline $\begin{array}{l}\text { Dosil, Díaz, Viñolas } \\
\text { y Díaz }\end{array}$ & 2012 & $\begin{array}{l}\text { Comprobar caracte- } \\
\text { rísticas (edad, género, } \\
\text { tipo de deporte, etc.) } \\
\text { de los deportistas con } \\
\text { TCA para poder inferir } \\
\text { causas de riesgo. }\end{array}$ & 308 sujetos & $\begin{array}{l}\text { Presión social } \\
\text { Presión del entrenador } \\
\text { Presión de los compañeros } \\
\text { Ser mujer } \\
\text { Deportes de riesgo }\end{array}$ & Comentarios reforzadores \\
\hline $\begin{array}{l}\text { De Sousa, De Sou- } \\
\text { sa y Caputo }\end{array}$ & 2016 & $\begin{array}{l}\text { Analizar las propieda- } \\
\text { des estadísticas de la } \\
\text { escalada DES }\end{array}$ & $\begin{array}{l}360 \text { atletas bra- } \\
\text { sileños }\end{array}$ & $\begin{array}{l}\text { Preocupación por la opinión de los } \\
\text { demás rivales } \\
\text { Creencia de relación del peso con } \\
\text { el rendimiento }\end{array}$ & \\
\hline Fabra y Casadó & 2014 & $\begin{array}{l}\text { Estudio de caso que } \\
\text { busca conocer y } \\
\text { comprender la rela- } \\
\text { ción que hay entre el } \\
\text { estilo de vida de una } \\
\text { joven deportista de } \\
\text { alto rendimiento y los } \\
\text { patrones funcionales } \\
\text { de salud }\end{array}$ & $\begin{array}{l}1 \text { patinadora ar- } \\
\text { tística en moda- } \\
\text { lidad grupal }\end{array}$ & $\begin{array}{l}\text { Estrés competitivo y bornout } \\
\text { Padecer una lesión } \\
\text { Dificultad para conciliar el sueño } \\
\text { Autoconcepto negativo } \\
\text { Falta de relaciones sociales exter- } \\
\text { nas al deporte }\end{array}$ & $\begin{array}{l}\text { Recursos de afrontamiento del estrés } \\
\text { Tener un adecuado cuidado de la salud } \\
\text { Nutrición equilibrada }\end{array}$ \\
\hline $\begin{array}{l}\text { Godoy, Díaz, Ra- } \\
\text { mírez, Navarrón y } \\
\text { Dosil }\end{array}$ & 2019 & $\begin{array}{l}\text { La búsqueda de facto- } \\
\text { res de riesgo en futbo- } \\
\text { listas en comparación } \\
\text { con otros deportes }\end{array}$ & 357 deportistas & $\begin{array}{l}\text { Ganancia de peso en periodos de } \\
\text { descanso } \\
\text { Comentarios de los compañeros }\end{array}$ & \\
\hline $\begin{array}{l}\text { Almena, Collazos y } \\
\text { Montero }\end{array}$ & 2016 & $\begin{array}{l}\text { Evaluar los factores de } \\
\text { riesgo de padecer TCA } \\
\text { en deportistas disca- } \\
\text { pacitados de alto nivel }\end{array}$ & $\begin{array}{l}81 \text { deportistas } \\
\text { de alto nivel con } \\
\text { discapacidad }\end{array}$ & $\begin{array}{l}\text { Pensar en quemar calorías mien- } \\
\text { tras hacen ejercicio. }\end{array}$ & $\begin{array}{l}\text { Autoconfianza alta } \\
\text { Discapacidades físicas que afectan al } \\
\text { movimiento }\end{array}$ \\
\hline Bär y Markser & 2013 & $\begin{array}{l}\text { Revisión de trastornos } \\
\text { mentales en deporte de } \\
\text { alto rendimiento }\end{array}$ & No especificado & Otros trastornos mentales & Un temprano diagnóstico \\
\hline $\begin{array}{l}\text { Díaz, Godoy-lz- } \\
\text { quierdo, Navarrón y } \\
\text { Ramírez }\end{array}$ & 2018 & $\begin{array}{l}\text { Revisión bibliográfica } \\
\text { para ver los factores } \\
\text { de riesgo y protección } \\
\text { con el fin de crear } \\
\text { unas pautas de pre- } \\
\text { vención de TCA en } \\
\text { futbolistas }\end{array}$ & No especificado & $\begin{array}{l}\text { Un aumento del rendimiento } \\
\text { Presión social } \\
\text { Comienzo temprano en el alto } \\
\text { rendimiento } \\
\text { Lesiones } \\
\text { Conductas alimentarias no salu- } \\
\text { dables } \\
\text { Cánones de belleza } \\
\text { Necesidad de control } \\
\text { Pesar a los deportistas regular- } \\
\text { mente }\end{array}$ & $\begin{array}{l}\text { Consecuencias en la salud a largo } \\
\text { plazo } \\
\text { Creencias sobre la importancia de la } \\
\text { salud } \\
\text { Contar con pautas nutricionales }\end{array}$ \\
\hline $\begin{array}{l}\text { Tan, Calitri Blood- } \\
\text { worth, y McNamee }\end{array}$ & 2016 & $\begin{array}{l}\text { Extraer los factores éti- } \\
\text { cos en el diagnóstico } \\
\text { de los TCA en gimnas- } \\
\text { tas de alto rendimiento }\end{array}$ & $\begin{array}{l}51 \text { gimnastas de } \\
4 \text { clubes diferen- } \\
\text { tes }\end{array}$ & $\begin{array}{l}\text { Comportamiento de los padres } \\
\text { desadaptativo } \\
\text { Tendencias de personalidad } \\
\text { Falta de satisfacción personal } \\
\text { Éxito temprano } \\
\text { Subida al alto rendimiento en edad } \\
\text { temprana } \\
\text { Rasgos depresivos }\end{array}$ & $\begin{array}{l}\text { Observar los cambios corporales } \\
\text { como normales } \\
\text { Apoyo psicológico por parte de los } \\
\text { entrenadores } \\
\text { Tener una buena autoestima }\end{array}$ \\
\hline
\end{tabular}




\section{Datos obtenidos}

En relación a la muestra de deportes, destacan los denominados de riesgo, y predomina una población mayoritariamente femenina, aunque cada vez más se empiezan a incluir la prevalencia de TCA en población de deportistas masculinos. Respecto a la distribución geográfica, los países más representados en la muestra de los artículos revisados son España, Estados Unidos, Brasil y otros países de Latinoamérica. Las investigaciones se centran en factores de riesgo más que en factores de protección: 86 factores extraídos corresponden a factores de riesgo y solo 36 corresponden a factores de protección.

Respecto a hábitos alimentarios inadecuados, los diferentes autores analizados han señalado que los deportistas tienen como un recurso disponible los vómitos, y el uso de laxantes y diuréticos para perder peso, como puede verse en el estudio de Engels et al. (2003) o el trabajo de Martínez et al. (2012) con remeros y luchadores, que concluyeron que un 52\% recurría frecuentemente al vómito para perder peso.

Respecto a la primera hipótesis de la investigación en relación factores que aumentan el riesgo de padecer TCA, encontramos una gran relevancia de aquellos relacionados con la presión social, de compañeros, del entrenador o del público (Martínez et al., 2012). Entre los desencadenantes principales destacados se encuentran: inicio de dieta, lesión y mayor periodo de competiciones. Y como mantenedores del problema: negación del trastorno, efectos positivos en el rendimiento, refuerzo social compañeros y entrenadores, sensación de ligereza, aumento de la sensación de control... (Díaz y Dosil, 2012).

La hipótesis 2 planteaba la relación el deporte con la imagen corporal. A este respecto se ha podido comprobar que realizar deportes en los que el tener un cuerpo delgado o un control del peso, aumenta aún más el riesgo de padecer TCA que en la población normal (Cano y Fernández, 2017). Sin embargo, en otros estudios hemos podido observar cómo en los deportes no considerados de riesgo como el fútbol o el voleibol también hay incidencia de este trastorno (Díaz et al., 2018; Frideres et al., 2014).

Los factores de personalidad del propio deportista también se relacionan con la mayor propensión a desarrollar un TCA, tal y como se afirma en el estudio de Godoy et al. (2019), aunque atletas con un gran impulso para la delgadez no siempre van ligados a la insatisfacción corporal (Bruin et al., 2011). Con ello se puede ver que las creencias, actitudes y comportamientos relacionados con la alimentación forman un conjunto complejo de factores que se relacionan entre sí para desencadenar, o no, en un trastorno.

Por otro lado, se encuentran los factores de protección obtenidos en la revisión, entre los que se encuentran la psicoeducación en TCA, los recursos de afrontamiento de cada deportista, una buena autoestima y autoconfianza o una adecuada y completa información nutricional (Martínez et al., 2012), tal y como se planteaba al inicio del documento en la hipótesis número 3.

Todo esto no resta importancia a los datos obtenidos en relación a la influencia que puede tener el entrenador y personas relacionadas con el mundo deportivo. Los datos demuestran que las personas del contexto deportivo ejercen una gran en el desarrollo o prevención del TCA en alto rendimiento, confirmando, por tanto, la cuarta hipótesis planteada para la presente revisión sistemática (Martínez et al., 2012).

\section{Discusión}

A la vista de los datos arrojados por la presente revisión, queda patente que las características del deportista y el deporte de alto rendimiento tienen influencia en la posibilidad de padecer un Trastorno de la Conducta Alimentaria en el presente o en el futuro. A pesar de ello, es importante tener en cuenta que esta relación de deporte de alto rendimiento y TCA no es una relación causal, pero sí relacional.

En esta línea Pellón (2002) ya realizó una revisión sistemática en el que introdujo el concepto de "anorexia por actividad", en el que se intenta aclarar si es el trastorno el que desencadena la práctica excesiva de ejercicio o si, por el contrario, es el ejercicio lo que lleva al trastorno. Podemos decir que se establece, por tanto, un círculo vicioso que relaciona el TCA con el deporte, donde el descenso de peso aumenta la 
motivación para la práctica de ejercicio físico y este disminuye el apetito (Cano y Fernández, 2017). Más allá de los deportes con mayor o menor riesgo, en el contexto general del alto rendimiento es importante reflexionar sobre el por qué de esta problemática: el deportista de alto rendimiento tiene ciertas exigencias (mantener un peso concreto, la orientación al resultado, o el régimen de competiciones) que pueden contribuir a establecer unos hábitos alimentarios inadecuados (Martínez et al., 2012).

Como factores de protección planteados, se ha demostrado en los resultados que una buena información y asesoramiento nutricional protege a los atletas de este trastorno, sin embargo, en la publicación de Dosil y Diaz (2003), se plasma la poca información que reciben los deportistas sobre aspectos alimentarios. Como consecuencia de esa desinformación los y las jóvenes deportistas están continuamente presionados para tener un "cuerpo ideal" para el rendimiento.

Tal y como mencionaban McArdle et al. (2015) y Lopes et al. (2009) el autoconcepto social establece un estigma asociado: cuando un deportista interioriza un rol fuerte en el ámbito deportivo es probable que extrapole esta identidad a las demás áreas de su vida, desembocando en comportamientos poco saludables para el individuo (Hernández-Mulero y Berengüí, 2016). Se puede ver en ello que preocupación por la percepción física del cuerpo se relaciona con la asociación que hizo también Festinger (citado en Bruin et al., 2011), observando la influencia de la comparación en el alto nivel deportivo. Estos resultados parecen estar de acuerdo con el concepto de "transitoriedad de satisfacción corporal" en los que los atletas a menudo experimentan diferentes niveles de satisfacción corporal en el deporte y la vida diaria (Bruin et al., 2011; Cardoso y García, 2009; Lopes et al., 2009).

Esto deja patente la relevancia de las intervenciones preventivas ya que, ha quedado ampliamente demostrado, que el conocimiento de los factores de riesgo y protección disminuye el riesgo a padecer un TCA (Díaz et al., 2018). Para una mejor eficacia, dichas intervenciones deben estar basadas en una comprensión de los factores de riesgo relevantes a nivel sociocultural (creencias sobre la alimentación, la dieta y el cuerpo de los atletas), conocimiento de los deportes y nivel contextual (presión de los entrenadores, estilo de entrenamiento, relaciones entre pares, patrones de alimentación familiar) y nivel personal (comparaciones con compañeros, perfeccionismo, creencias y prácticas en relación con la comida y el peso). En este campo los entrenadores, debido a su contacto directo y continuo con los atletas, deberían considerarse un elemento clave en el manejo del TCA, incluida la reducción de riesgos y la detección temprana e intervención temprana.

\section{Áreas de mejora y futuras líneas de investigación}

En relación a los factores de riesgo y de protección en TCA existen pocas investigaciones que se centren en la prevención y tratamiento de las mismas, dentro del contexto del alto rendimiento deportivo (Hernández-Mulero y Berengüí, 2016). Además, la investigación hasta la fecha se focaliza en los factores de riesgo para los TCA, pero los factores protectores son igualmente relevantes, los cuáles han sido mayoritariamente ignorados en la investigación. La investigación futura debería conceder la misma importancia a los factores de riesgo y de protección (Díaz et al., 2018; Hausenblas y Carron, 1999).

A pesar de que existen ciertos deportes de riesgo y otros que se consideran de menos riesgo, con el análisis de los datos obtenidos, se ha podido ver un riesgo creciente en grupo de menor riesgo, apelando a la necesidad de realizar más estudios de este grupo, ya que no se clarifica que realmente tenga ciertamente una menor incidencia respecto a los deportes de mayor riesgo (Cano y Fernández, 2017).

Es necesario añadir que la mayoría de estudios sobre TCA en el deporte son realizados con mujeres, especialmente en disciplinas de estética. Por lo tanto, sería interesante estudiar con mayor profundidad este campo en futuras investigaciones que avalen los resultados aquí obtenidos (Hernández-Mulero y Berengüí, 2016).

El verdadero desafío en deportistas de alto rendimiento radica diferenciar entre actitudes y comportamientos extremos que, aunque aparentemente parezcan patológicos, se racionalizan en el contexto deportivo, y son reflejados y respaldados por él (Martínez et al., 2012; Tan et al., 2016). Por lo tanto, en relación a instrumentos utilizados como el DES (Disordered Eating in Sports Scale), es extremadamente 
importante diseñar instrumentos con elementos específicos que aborden el entorno de los deportes para analizar de manera fiable la frecuencia de las conductas alimentarias desorganizadas entre los atletas (De Sousa et al., 2016).

\section{Aplicaciones prácticas}

Debido a la demostrada influencia de las falsas creencias en los Trastornos de la Conducta Alimentaria en los estudios revisados (Díaz et al., 2018; Martínez et al., 2012), como primer paso a realizar se destaca la necesidad de desmentir ciertos mitos y creencias erróneas sobre el peso y el rendimiento deportivo, y dar información de calidad,

Como segundo paso se debe tener en cuenta la educación nutricional, realizar estudios dietéticos personalizados, teniendo en cuenta la condición física del deportista, su nivel de entrenamiento y ritmo de vida (Díaz y Dosil. 2012; Lopes et al., 2009).

Debido a la gran influencia de la familia y entrenadores (Hernández-Mulero y Berengüí, 2006; Martínez et al., 2012), el tercer ámbito de trabajo se debe centrar en una formación y educación óptimas de los entrenadores, familiares y entorno para que sean capaces de realizar la prevención y la detección temprana.

Como cuarto paso, en caso de detectar un TCA debe iniciarse la terapia cuanto antes, la cual deberá llevarse a cabo a través de un equipo multidisciplinar (médicos, nutricionistas, psicólogos y/o psiquiatras) (Frideres et al., 2014).

Tal y como decían Hernández-Mulero y Berengüí (2016), otro aspecto de vital importancia de acuerdo con los datos analizados es evitar que los deportistas desarrollen niveles desadaptativos de identidad deportiva. Por ello, es necesario fomentar que el deportista disponga de otros intereses e inquietudes al margen del deporte, así como propiciar momentos y periodos de desconexión.

En definitiva, no hay que olvidar que la eficacia de los programas preventivos se ha documentado en atletas, pero se necesitan los esfuerzos combinados de psicólogos, sanitarios (médicos, enfermeros, nutricionistas) y profesionales del deporte para mejorar el tratamiento y la prevención de estas enfermedades (Bär y Markser, 2013).

\section{Referencias}

* Almena, F. J., Collazos, J. F. y Montero, M. D. (2016). Spanish high level handicapped sportsmen and eating disorders: are they at risk? Journal of Negative and No Positive Results: JONNPR, 1(7), 262-267.

* Bär K. y Markser V. (2013). Sport specificity of mental disorders: the issue of sport psychiatry. European Archives of Psychiatry and Clinical Neuroscience, 263(2), 205-210. https://doi.org/10.1007/s00406-0130458-4

* Bruin, A., Oudejans, R. R., Bakker, F. C. y Woertman, L. (2011). Contextual body image and athletes' disordered eating: The contribution of athletic body image to disordered eating in high performance women athletes. European Eating Disorders Review, 19, 201-215.

* Cano, M. y Fernández, A. (2017). Anorexia nerviosa y ejercicio físico. Trastornos de la Conducta Alimentaria, 25, 2795-2809.

* De la Fuente, A. (2018). Height categories as a healthier alternative to weight categories in Taekwondo competition. Revista de Artes Marciales Asiáticas, 13(1), 53-60.

* De Sá, A., Aliete Dos Santos, D. y Simões, R. (2017). La conducta alimentaria y factores relacionados se asocian con un riesgo de trastornos alimentarios en las mujeres físicamente activas. Nutrición Clínica y Dietética Hospitalaria, 37 (1), 141-148.

* De Sousa, L., De Sousa, S. y Caputo, M. E. (2016). Psychometric Analysis of Disordered Eating in Sports Scale (DES). Paidéia, 26(64), 171-180. https://doi.org/10.1590/1982-43272664201603 
* Díaz, I. y Dosil, J. (2012). Detección y prevención de los trastornos de la conducta alimentaria en el deporte (Tesis Doctoral). Universidad de Vigo.

* Díaz, I., Godoy-Izquierdo, D., Navarrón, E., Ramírez, M. J. y Dosil, J. (2018). Eating disorders in sports and football: An updated review. Cuadernos de Psicología del Deporte, 2(18), 45-56.

Dosil-Díaz, J. (2003). Trastornos de alimentación en el deporte. Editorial deportiva Wanceulen.

* Dosil, J., Díaz, I., Viñolas, A. y Díaz, O. (2012). Prevención y detección de los trastornos de alimentación en deportistas de alto rendimiento (CAR, CEARE y CTD). Cuadernos de Psicología del Deporte, 12(1), 163-166.

El Ghoch, M., Soave, F., Calugi, S. y Dalle Grave, R. (2013). Eating disorders, physical fitness and sport performance: a systematic review. Nutrients, 5(12), 5140-5160. https://doi.org/10.3390/nu5125140

Engels, S., Johnson, C., Power, P., Crosby, R., Wonderlich, S., Witrock, D. y Mitchell, J. (2003). Predictors of disordered eating in a sample of elite Division I college athletes. Eating Behaviors, 4, 333-343.

*Fabra, J. y Casadó, L. (2014). Relación entre el estilo de vida de una joven deportista de alto rendimiento y los patrones funcionales de salud de Marjory Gordon. Cultura de los cuidados, 18(38), 107-117. https:// doi.org/10.7184/cuid.2014.38.13

Franchini, E., Brito, C. y Artioli, G. (2012). Weight loss in combat sports: Physiological, mancpsychological and perfore effects. Journal of the International Society of Sports Nutrition, 9, Artículo e52. https://doi. org/10.1186/1550-2783-9-52

* Frideres, J., Visiedo, A. y Palao, J. (2014). Efecto de la edad sobre la percepción de los factores de riesgo de los trastornos de la conducta alimentaria en jugadoras senior profesionales de voleibol. Revista Euroamericana de Ciencias del Deporte, 3(1), 27-32.

*Godoy, D., Díaz, I., Ramírez, M. J., Navarrón, E. y Dosil, J. (2019). Risk for eating disorders in "high"- and "low"-risk sports and football (soccer): A profile analysis with clustering techniques. Revista de Psicología del Deporte, 28(2), 117-126.

* Goltz, F. R., Stenzel, L. M. y Schneider, C. D. (2013). Disordered eating behaviors and body image in male athletes. Revista Brasileira de Psiquiatría, 35, 237-242.

Hausenblas, H. A. y Carron, A.V. (1999). Eating disorders indices and athletes: an integration. Journal of Sport and Exercise Psychology, 21, 230-258.

* Hernández-Mulero, N. y Berengüí, R. (2016). Identidad deportiva y Trastornos de la Conducta Alimentaria: estudio preliminar en deportistas de competición. Cuadernos de Psicología del Deporte, 16(2), 37-44.

* Lopes, J. L., Zotto, H., Fiorese, L., Cardoso, A. y Garcia, P. (2009). Distúrbios de atitudes alimentares e distorção da imagem corporal no contexto competitivo da ginástica rítmica. Revista Brasileira de Medicina do Esporte, 15(6), 410-414. https://doi.org/10.1590/S1517-86922009000700001

* Loucks, A. (2014). La tríada de la atleta: un fenómeno metabólico. Revista de Ciencias del Ejercicio y la Salud, 12(1), 1-24.

* Martínez, A., Pérez, P., Santín, L. y Arrue, M (2018). Perfect body (1997): trastornos alimentarios en deportistas de élite, factores de riesgo y sintomatología. Revista de Medicina y Cine, 14(4), 237-243.

* Martínez, J. M., Urdampilleta, A., Micó, L. y Soriano, J. M. (2012). Aspectos psicológicos y sociológicos en la alimentación de los deportistas. Cuadernos de Psicología del Deporte, 12(2), 39-48.

Mateos, M., Torregrosa, M. y Cruz, J. (2010). Evaluation of a career assistance programme for elite athletes: satisfaction levels and exploration of career decision making and athletic-identity. Kinesiologia Slovenica, $16,30-43$.

* McArdle, S., Meade, M. y Moore, P., (2016). Exploring attitudes toward eating disorders among elite athlete support personnel. Medicine Science Sports, 26, 1117-1127.

Packard, A. N. (2010). Conflicto de identidades: la identidad deportiva versus la identidad nacional en Barcelona. Apunts. Educación Física y Deportes, 100, 7-13.

Pellón, M. T. (2002). Anorexia por actividad: una revisión teórica y experimental. International Journal Of Psycology and Psycological Therapy, 131-145.

Pérez, G., Rodríguez, F., Esteve, E., Larraburu, I., Font, J. y Pons, V. (1992). Prevalencia de trastornos de la conducta alimentaria en deportistas. Revista Psicología del Deporte, 1(1), 5-16. 
* Prnjak, K., Jukic, I. y Tufano, J. J. (2019). Perfectionism, Body Satisfaction and Dieting in Athletes: The Role of Gender and Sport Type. Sports, 7, 181.

Sundgot-Borgen J. y Torstveit, M. K. (2004). Prevalence of eating disorders in elite athletes is higher than in the general population. Clinical Journal of Sport Medicine. 14(1), 25-32.

* Tan, J., Calitri R., Bloodworth A. y McNamee M. J. (2016). Understanding Eating Disorders in Elite Gymnastics. Ethical and Conceptual Challenges Tan Clinics in Sports Medicine, 35(2), 275-292.

*Thiemann, P., Legenbauer, T., Vocks, S., Platen, P., Auyeung, B. y Herpertz, S. (2015). Eating Disorders and Their Putative Risk Factors Among Female German Professional Athletes. European Eating Disorders Review, 23(4), 269-276. https://doi.org/10.1002/erv.2360

Artículo recibido: $28 / 05 / 2020$

Artículo aceptado: 25/03/2021 